\begin{tabular}{|c|l|}
\hline Title & N-player mosquito net game: Individual and social rationality in the misuse of insecticide treated nets \\
\hline Author(s) & Honjo, Keita; Satake, A kiko \\
\hline Citation & $\begin{array}{l}\text { Journal of Theoretical Biology, 342,39-46 } \\
\text { https://doi.org/40.1016/.jtbi.2013.11.001 }\end{array}$ \\
\hline Issue Date & 2014.02-07 \\
\hline Doc URL & http://hdl.handle.net/2115/54872 \\
\hline Type & article (author version) \\
\hline File Information & Honjo_JTB2014Manuscript.pdf \\
\hline
\end{tabular}

Instructions for use 


\title{
N-player mosquito net game: Individual and social rationality in the misuse of insecticide-treated nets
}

\author{
Keita Honjo ${ }^{\mathrm{a}, *}$, Akiko Satake ${ }^{\mathrm{b}}$ \\ ${ }^{a}$ Graduate School of Environmental Science, Hokkaido University, N10W5 Sapporo, \\ Hokkaido 060-0810, Japan \\ ${ }^{b}$ Faculty of Environmental Earth Science, Hokkaido University, N10W5 Sapporo, \\ Hokkaido 060-0810, Japan
}

Keywords: Malaria, poverty, game theory, decision making

\begin{abstract}
Many governmental and non-governmental organizations have distributed insecticide-treated nets (ITNs) to malaria endemic areas, which contributed to the reduction of malaria deaths. However, some people in malaria endemic areas used ITNs for alternative purposes such as fishery and agriculture. It is unclear why people threatened by malaria misuse ITNs. Here we develop a $N$-player mosquito net game, and theoretically show that the misuse of ITNs might be underpinned by individual and social rationality. In the mosquito net game, each player uses ITNs for malaria prevention or alternative purposes. The proper ITN use decreases the probability of malaria infection, while the improper ITN use increases the player's labor productivity. Each player's expected payoff is influenced by other players' strategies. We found that the misuse of ITNs can be a Pareto efficient Nash equilibrium. The
\end{abstract}

\footnotetext{
*Corresponding author

Email address: keita_honjo@ees.hokudai.ac.jp (Keita Honjo)
} 
maximum number of players using ITNs for malaria prevention is limited by insecticidal effectiveness of ITNs and extra income from ITN misuse. Furthermore, we found that players in a low-income community are attracted to the misuse of ITNs even if the probability of malaria infection is high. Introduction of a tax on ITN misuse was shown to be effective to motivate the players to use ITNs for malaria prevention. Our results demonstrate that understanding decision making of people in malaria endemic areas is essential to design more effective malaria control programs.

\section{Introduction}

To eliminate malaria, many governmental and non-governmental organizations have distributed insecticide-treated nets (ITNs) to malaria endemic areas free of charge or at subsidized prices [17]. ITNs kill mosquitoes which contact the nets, and protect people sleeping inside the nets from mosquito bites. Several authors have reported that the distribution of ITNs contributed to the reduction of malaria deaths. In Kenya, an increase in ITN coverage from $7 \%$ to $67 \%$ resulted in a $44 \%$ reduction of mortality caused by malaria [6]. ITN use was especially effective for protection of children under five years old who are vulnerable to malaria [11]. In Vanuatu in the South Pacific Ocean, the number of malaria cases was reduced by at least $50 \%$ when at most $20 \%$ of the population at risk was covered by ITNs [2]

Nevertheless, it has been reported that distributed ITNs were misused for fishery and agriculture. In major fishing villages in western Kenya, a considerable number of ITNs were used for catching and drying fish [14]. In Ethiopia, some people used ITNs for drying grain, tying cattle to a tree, and 
tablecloths, etc. [1]. Similar misuses have also been observed in Timor-Leste in Southeast Asia [12]. These ITN misuses would reduce public health benefits expected from ITN distribution. Thus, understanding how people make decision about ITN use is necessary for the success of malaria control programs. However, little is known about decision making behind ITN misuse, and it remains unclear why people threatened by malaria misuse ITNs.

We have shown that game theory, which describes conflicts of interest behind human behaviors, is a useful tool to address the ITN-misuse problem. A two-player non-cooperative game (called a mosquito net game) theoretically showed that ITN misuse can be an individually and socially rational strategy in low-income areas [8]. Furthermore, we found that the misuse of ITNs is associated with free rider problems which emerge in the voluntary provision of public goods $[13,21]$ and the shared use of ecosystem services [20]. ITN use for malaria prevention decreases mosquito population size around the community, and indirectly protects the whole community from malaria $[9,7,10]$. This community effect plays a role similar to public goods. By free riding on the community effect provided by proper ITN users, improper ITN users can decrease the infection probability without abandoning extra income from ITN misuse.

This paper extends the two-player mosquito net game into an $N$-player game. The $N$-player mosquito net game covers a wider range of situations than the two-player game, and enables us to evaluate how human community size influences decision making of ITN users. We also evaluate the potential impact of imposing a tax on ITN misuse as an incentive to promote ITN use for malaria prevention. 


\section{Structure of the game}

The $N$-player mosquito net game is a one-shot normal form game with the following structure: each player, denoted by $i \in\{1,2, \ldots, N\}$, has the set of pure strategies $\{\mathrm{T}, \mathrm{F}\}$. A player with strategy $\mathrm{T}$ uses ITNs for malaria prevention. A player with strategy $\mathrm{F}$ misuses ITNs for economic activities such as fishery and agriculture. The profile of strategies for the $i$-th player with strategy $\sigma_{i} \in\{\mathrm{T}, \mathrm{F}\}$ is denoted as $\left(\sigma_{i}, m_{-i}\right)$. Note that $m_{-i} \in\{0,1, \ldots, N-1\}$ is the number of players with strategy $\mathrm{T}$ except the $i$-th player.

Let $P \in[0,1]$ be the probability of malaria infection for each player with no protection from mosquito bites. The infection probability of the $i$-th player at a profile $\left(\sigma_{i}, m_{-i}\right)$ is given by

$$
p_{i}\left(\sigma_{i}, m_{-i}\right):=\left\{\begin{array}{ll}
\alpha_{1} \alpha_{2}^{m_{-i}+1} P & \left(\sigma_{i}=\mathrm{T}\right) \\
\alpha_{2}^{m_{-i}} P & \left(\sigma_{i}=\mathrm{F}\right)
\end{array},\right.
$$

where $\alpha_{1} \in(0,1)$ and $\alpha_{2} \in(0,1)$ are the parameters representing individual and (per capita) community effects of ITNs, respectively. Note that lower values of $\alpha_{1}$ and $\alpha_{2}$ mean stronger individual and community effects, respectively. The individual effect decreases the infection probability for each player with strategy $\mathrm{T}$ by physically protecting it from mosquito bites $[11,6,2]$. On the contrary, the community effect decreases every player's infection probability regardless of strategy. Properly-used ITNs repel or kill mosquitoes when they approach or contact the nets, likely reducing the entomological risk of malaria infection $[9,7,10]$. We assume that when $m$-players, $m \in\{0,1, \ldots, N\}$, use ITNs for malaria prevention, the aggregate commu- 
nity effect equals $\alpha_{2}^{m}$. The power functional form is necessary to simplify equilibrium analysis of the game. An alternative functional form is shown in Appendix A.

ITNs can have the misuse effect which increases labor productivity of each player with strategy F. Let $L \in[0, \infty)$ (e.g., \$/capita) be labor productivity of each player with strategy $\mathrm{T}$. The labor productivity of the $i$-th player at a profile $\left(\sigma_{i}, m_{-i}\right)$ is given by

$$
l_{i}\left(\sigma_{i}, m_{-i}\right):=\left\{\begin{array}{ll}
L & \left(\sigma_{i}=\mathrm{T}\right) \\
\beta L & \left(\sigma_{i}=\mathrm{F}\right)
\end{array},\right.
$$

where $\beta \in(1, \infty)$ is the parameter representing the misuse effect. A higher value of $\beta$ means a stronger misuse effect.

Assume that any player infected with malaria cannot work and has zero labor productivity $(L=0)$. The expected payoff for the $i$-th player is calculated as

$$
\begin{aligned}
U_{i}\left(\sigma_{i}, m_{-i}\right) & =p_{i}\left(\sigma_{i}, m_{-i}\right) \times 0+\left(1-p_{i}\left(\sigma_{i}, m_{-i}\right)\right) \times l_{i}\left(\sigma_{i}, m_{-i}\right) \\
& = \begin{cases}\left(1-\alpha_{1} \alpha_{2}^{m_{-i}+1} P\right) L & \left(\sigma_{i}=\mathrm{T}\right) \\
\left(1-\alpha_{2}^{m_{-i}} P\right) \beta L & \left(\sigma_{i}=\mathrm{F}\right)\end{cases}
\end{aligned}
$$

This is the payoff function for the $N$-player mosquito net game.

In the mosquito net game, the community effect plays a role similar to public goods, and gives the players an incentive to be free riders. In economics, free riders are people who use public goods without paying any cost to the providers $[13,21]$. If there is no community effect $\left(\alpha_{2}=1\right)$, each player's payoff is independent of other players' strategies, and every player 
chooses the same strategy (called the all-F or all-T profile). If there is a community effect $\alpha_{2} \in(0,1)$, players with strategy $\mathrm{F}$ coexist with players with strategy $\mathrm{T}$ (called the free-rider profile). When a free-rider profile is chosen by the players, alternative ITN users free ride on the community effect provided by those individuals who use their ITN for malaria protection.

\section{Nash and Pareto equilibria}

Here we investigate Nash and Pareto equilibria of the $N$-player mosquito net game. The Nash and Pareto equilibria are criteria for individual and social rationality, respectively. The Nash equilibrium is not always Pareto efficient (a Pareto equilibrium). When each player's strategy satisfies both individual and social rationality, the profile of strategies is a Pareto efficient Nash equilibrium (PNE). The Pareto "inefficient" Nash equilibrium is called a social dilemma (SD), which is illustrated by the well known prisoner's dilemma $[4,21]$. When the players are attracted to a social dilemma, an individually rational strategy results in a socially irrational payoff.

\subsection{Nash equilibrium}

The Nash equilibrium is the set of profiles where any player has no incentive to change its strategy $[15,16]$. The $N$-player mosquito net game can have three types of Nash equilibria: all-F, free-rider, and all-T. At the all-F (all-T) Nash equilibrium, each player has the strategy F (strategy T), which is the best response to other players' strategies. At the free-rider Nash equilibrium, $k$-players have the strategy $\mathrm{T}$ and other players have the strategy $\mathrm{F}$. The number $k \in\{1,2, \ldots, N-1\}$ is called the degree of the free-rider Nash equilibrium. Conditions for the Nash equilibria are derived as follows. 
First, the all-F profile is a Nash equilibrium if $U_{i}(\mathrm{~F}, 0) \geq U_{i}(\mathrm{~T}, 0)$. Solving this inequality with respect to $P$ gives

$$
P \in\left[0, P_{0}\right], \quad P_{0}:=\frac{\beta-1}{\beta-\alpha_{1} \alpha_{2}} .
$$

Second, the $k$-th degree free-rider Nash equilibrium requires two inequalities. For the $i$-th player with strategy $\mathrm{T}, U_{i}(\mathrm{~T}, k-1) \geq U_{i}(\mathrm{~F}, k-1)$. For the $j$-th player with strategy $\mathrm{F}, U_{j}(\mathrm{~F}, k) \geq U_{j}(\mathrm{~T}, k)$. From these inequalities,

$$
P \in\left[P_{k-1}, P_{k}\right] \cap[0,1], \quad P_{k}:=\frac{P_{0}}{\alpha_{2}^{k}} .
$$

Third, the all-T profile is a Nash equilibrium if $U_{i}(\mathrm{~T}, N-1) \geq U_{i}(\mathrm{~F}, N-$ 1). From this inequality,

$$
P \in\left[P_{N-1}, 1\right], \quad P_{N-1}:=\frac{P_{0}}{\alpha_{2}^{N-1}} .
$$

\subsection{Pareto equilibrium}

The Pareto equilibrium is the set of Pareto efficient profiles where no player can increase its payoff without decreasing other players' payoffs. Let $\mathbf{S}:=\left\{\left(\sigma_{1}, \sigma_{2}, \ldots, \sigma_{N}\right) \mid \sigma_{i} \in\{\mathrm{T}, \mathrm{F}\}\right\}$ be the universal set of profiles. A profile $s^{*} \in \mathbf{S}$ is Pareto efficient (a Pareto equilibrium) if there is no profile $s \in \mathbf{S}$ such that $U_{i}(s) \geq U_{i}\left(s^{*}\right)$ for any player $i$ and $U_{j}(s)>U_{j}\left(s^{*}\right)$ for a player $j[19,5]$. The Pareto efficiency is desirable for the whole community in the sense that there is no possibility to increase all players' payoffs simultaneously [21].

A Pareto equilibrium is the profile to which all profiles are Pareto inferior. Let $\mathbf{u}(s):=\left(U_{1}(s), U_{2}(s), \ldots, U_{N}(s)\right)$ be the payoff vector at a profile $s . \mathbf{u}(s)$ is Pareto superior to the payoff vector $\mathbf{u}\left(s^{\prime}\right)$ at a profile $s^{\prime} \in \mathbf{S}$ if

$$
\mathbf{u}(s) \in \mathbf{P}\left(s^{\prime}\right), \quad \mathbf{P}\left(s^{\prime}\right):=\left(\prod_{i=1}^{N}\left[U_{i}\left(s^{\prime}\right), \infty\right)\right) \backslash\left\{\mathbf{u}\left(s^{\prime}\right)\right\},
$$


where $\mathbf{P}\left(s^{\prime}\right)$ indicates the Pareto superior region to $\mathbf{u}\left(s^{\prime}\right)$ (see Fig. 1a for $N=2$ ). By moving from $\mathbf{u}\left(s^{\prime}\right)$ to $\mathbf{P}\left(s^{\prime}\right)$, at least one player can increase its payoff without decreasing other players' payoffs. The complement of $\mathbf{P}\left(s^{\prime}\right)$ in the whole region $[0, \infty)^{N}$ is the Pareto inferior region

$$
\mathbf{P}^{C}\left(s^{\prime}\right):=[0, \infty)^{N} \backslash \mathbf{P}\left(s^{\prime}\right)
$$

(see Fig. $1 \mathrm{~b}$ for $N=2$ ). The move from $\mathbf{u}\left(s^{\prime}\right)$ to $\mathbf{P}^{C}\left(s^{\prime}\right)$ maintains the payoff vector or decreases at least one player's payoff.

A payoff vector $\mathbf{u}\left(s^{*}\right)$ is Pareto efficient (a Pareto equilibrium) if

$$
\mathbf{u}(s) \in \mathbf{P}^{C}\left(s^{*}\right), \forall s \in \mathbf{S} .
$$

It follows that all profiles are Pareto inferior to a Pareto equilibrium. The $N$-player mosquito net game can have all-T, free-rider, and all-F Pareto equilibria. Conditions for the Pareto equilibria are derived as follows.

\subsubsection{All-F Pareto equilibrium}

Let $\mathbf{S}_{m} \subset \mathbf{S}, m \in\{0,1, \ldots, N\}$ be the set containing all profiles at which $m$-players have the strategy $\mathrm{T}$. The all-F profile $s_{0}=(\mathrm{F}, 0)$, which is the unique element of $\mathbf{S}_{0}$, is a Pareto equilibrium if $\mathbf{u}(s) \in \mathbf{P}^{C}\left(s_{0}\right)$ for all $s \in \mathbf{S}$. Here we demonstrate that all profiles are Pareto inferior to the all-F profile when $P \in\left[0, P_{0}^{*}\right], P_{0}^{*}:=(\beta-1) /\left(\beta-\alpha_{1} \alpha_{2}^{N}\right)$. Under this condition, a shift from the all-F to any profile maintains the payoff vector or decreases at least one player's payoff. When the players shift from the all-F to any profile, the $i$-th player's profile $\left(\sigma_{i}, m_{-i}\right), m_{-i} \in\{0,1, \ldots, N-1\}$ can change in four ways (Table 1 ). 
First, any profile is Pareto inferior to itself due to the definition (8). When the players shift from the all-F profile to itself, every player is type S1, and the payoff vector is maintained. Hence

$$
\mathbf{u}\left(s_{0}\right) \in \mathbf{P}^{C}\left(s_{0}\right)
$$

Second, the all-T profile $s_{N}=(\mathrm{T}, N-1)$, which is the unique element of $\mathbf{S}_{N}$, is Pareto inferior to the all-F profile if $P \in\left[0, P_{0}^{*}\right]$. When the players shift from the all-F to all-T profile, every player is type S3. If $U_{i}(\mathrm{~F}, 0) \geq$ $U_{i}(\mathrm{~T}, N-1)$, the profile shift maintains the payoff vector or decreases every player's payoff. From the inequality,

$$
\mathbf{u}\left(s_{N}\right) \in \mathbf{P}^{C}\left(s_{0}\right) \leftrightarrow P \in\left[0, P_{0}^{*}\right], \quad P_{0}^{*}=\frac{\beta-1}{\beta-\alpha_{1} \alpha_{2}^{N}} .
$$

Third, every degree free-rider profile is Pareto inferior to the all-F profile under the condition (11). The set $\mathbf{S}_{k}$ contains ${ }_{N} C_{k}$ profiles with degree $k \in \mathbf{K}:=\{1,2, \ldots, N-1\}$, which are abbreviated as $(\mathrm{T}, k-1)$ or $(\mathrm{F}, k)$ depending on the $i$-th player's strategy. When the players shift from the all-F to a $k$-th degree free-rider profile $s_{k} \in \mathbf{S}_{k}, k$-players are type $\mathrm{S} 2 \mathrm{a}$, and other players are type S2b. Since $U_{i}(\mathrm{~F}, 0)<U_{i}(\mathrm{~F}, k)$, the inequality $U_{i}(\mathrm{~F}, 0)>U_{i}(\mathrm{~T}, k-1)$ is required for the Pareto inferiority of $s_{k}$ to $s_{0}$. Under the condition (11), the inequality $U_{i}(\mathrm{~F}, 0) \geq U_{i}(\mathrm{~T}, N-1)>U_{i}(\mathrm{~T}, k-1)$ holds. Hence

$$
P \in\left[0, P_{0}^{*}\right] \rightarrow \mathbf{u}\left(s_{k}\right) \in \mathbf{P}^{C}\left(s_{0}\right), \forall k \in \mathbf{K}, \forall s_{k} \in \mathbf{S}_{k} .
$$

All profiles are Pareto inferior to the all-F profile under the condition (11). 


\subsubsection{Free-rider Pareto equilibria}

A $k$-th degree free-rider profile $s_{k}$ is a Pareto equilibrium if

$$
\mathbf{u}(s) \in \mathbf{P}^{C}\left(s_{k}\right), \forall k \in \mathbf{K}, \forall\left(s, s_{k}\right) \in \mathbf{S} \times \mathbf{S}_{k} .
$$

Here we demonstrate that all profiles are Pareto inferior to any $k$-th degree free-rider profile when $P \in\left[0, P_{k}^{*}\right) \cap[0,1], P_{k}^{*}:=(\beta-1) /\left(\alpha_{2}^{k} \beta-\alpha_{1} \alpha_{2}^{N}\right)$. Under this condition, a shift from $s_{k}$ to any profile maintains the payoff vector or decreases at least one player's payoff. When the players shift from $s_{k}$ to any profile, the $i$-th player's profile can change in 16 ways (Table 2).

First, free-rider profiles with the same degree are Pareto inferior to each other. When the players shift from $s_{k}$ to $s_{k}^{\prime} \in \mathbf{S}_{k}$, the number of players switching from $\mathrm{T}$ to $\mathrm{F}$ equals that switching from $\mathrm{F}$ to $\mathrm{T}$. If a type-S4b player increases its payoff with the profile shift, there exists a type-S4c player which decreases its payoff. If every player is type $\mathrm{S} 4 \mathrm{a}$ or $\mathrm{S} 4 \mathrm{~d}$, the payoff vector is maintained. The shift from $s_{k}$ to $s_{k}^{\prime}$ maintains the payoff vector or decreases at least one player's payoff:

$$
\mathbf{u}\left(s_{k}^{\prime}\right) \in \mathbf{P}^{C}\left(s_{k}\right), \forall k \in \mathbf{K}, \forall\left(s_{k}, s_{k}^{\prime}\right) \in \mathbf{S}_{k} \times \mathbf{S}_{k} .
$$

Second, the all-T profile is Pareto inferior to any $k$-th degree free-rider profile if $P \in\left[0, P_{k}^{*}\right) \cap[0,1]$. When the players shift from $s_{k}$ to $s_{N}, k$ players are type S8a, and other players are type S8b. Since $U_{i}(\mathrm{~T}, k-1)<$ $U_{i}(\mathrm{~T}, N-1)$, the inequality $U_{i}(\mathrm{~F}, k)>U_{i}(\mathrm{~T}, N-1)$ is required for the Pareto inferiority of $s_{N}$ to $s_{k}$. From the inequality,

$$
\begin{gathered}
\mathbf{u}\left(s_{N}\right) \in \mathbf{P}^{C}\left(s_{k}\right) \leftrightarrow P \in\left[0, P_{k}^{*}\right) \cap[0,1], \forall k \in \mathbf{K}, \forall s_{k} \in \mathbf{S}_{k}, \\
P_{k}^{*}=\frac{\beta-1}{\alpha_{2}^{k} \beta-\alpha_{1} \alpha_{2}^{N}} .
\end{gathered}
$$


Third, the all-F profile is Pareto inferior to every degree free-rider profile. When the players shift from $s_{k}$ to $s_{0}, k$-players are type S5a, and other players are type S5b. Since $U_{i}(\mathrm{~F}, k)>U_{i}(\mathrm{~F}, 0)$,

$$
\mathbf{u}\left(s_{0}\right) \in \mathbf{P}^{C}\left(s_{k}\right), \forall k \in \mathbf{K}, \forall s_{k} \in \mathbf{S}_{k} .
$$

Fourth, under the condition (15), free-rider profiles with degrees lower than $k$ are Pareto inferior to any $k$-th degree free-rider profile. When the players shift from $s_{k}$ to $s_{k_{0}} \in \mathbf{S}_{k_{0}}$ with a lower degree $k_{0} \in \mathbf{K}_{0}:=\{1,2, \ldots, k-$ 1 , each player is type S6a, S6b, S6c, or S6d. At least one player is type S6a, S6c, or S6d. For the types S6a and S6d, the inequalities $U_{i}(\mathrm{~T}, k-1)>$ $U_{i}\left(\mathrm{~T}, k_{0}-1\right)$ and $U_{i}(\mathrm{~F}, k)>U_{i}\left(\mathrm{~F}, k_{0}\right)$ hold, respectively. For the type S6c, the inequality $U_{i}(\mathrm{~F}, k)>U_{i}(\mathrm{~T}, N-1)>U_{i}\left(\mathrm{~T}, k_{0}-1\right)$ holds under the condition (15). The shift from $s_{k}$ to $s_{k_{0}}$ decreases at least one player's payoff if the all-T profile $s_{N}$ is Pareto inferior to $s_{k}$ :

$$
\begin{aligned}
& P \in\left[0, P_{k}^{*}\right) \cap[0,1] \rightarrow \mathbf{u}\left(s_{k_{0}}\right) \in \mathbf{P}^{C}\left(s_{k}\right), \\
& \forall\left(k, k_{0}\right) \in \mathbf{K} \times \mathbf{K}_{0}, \forall\left(s_{k}, s_{k_{0}}\right) \in \mathbf{S}_{k} \times \mathbf{S}_{k_{0}}
\end{aligned}
$$

Fifth, under the condition (15), free-rider profiles with degrees higher than $k$ are Pareto inferior to any $k$-th degree free-rider profile. When the players shift from $s_{k}$ to $s_{k_{1}} \in \mathbf{S}_{k_{1}}$ with a higher degree $k_{1} \in \mathbf{K}_{1}:=\{k, k+1, \ldots, n-1\}$, each player is type S7a, S7b, S7c, or S7d. Since the number of players with strategy $\mathrm{T}$ at $s_{k_{1}}$ is greater than that at $s_{k}$, at least one player is type $\mathrm{S} 7 \mathrm{c}$. Since the inequality $U_{i}(\mathrm{~F}, k)>U_{i}(\mathrm{~T}, N-1)>U_{i}\left(\mathrm{~T}, k_{1}-1\right)$ holds under the condition (15), the type-S7c player decreases its payoff if the all-T profile $s_{N}$ 
is Pareto inferior to $s_{k}$ :

$$
\begin{aligned}
& P \in\left[0, P_{k}^{*}\right) \cap[0,1] \rightarrow \mathbf{u}\left(s_{k_{1}}\right) \in \mathbf{P}^{C}\left(s_{k}\right), \\
& \forall\left(k, k_{1}\right) \in \mathbf{K} \times \mathbf{K}_{1}, \forall\left(s_{k}, s_{k_{1}}\right) \in \mathbf{S}_{k} \times \mathbf{S}_{k_{1}} .
\end{aligned}
$$

All profiles are Pareto inferior to any $k$-th degree free-rider profile under the condition (15).

\subsubsection{All-T Pareto equilibrium}

The all-T profile is a Pareto equilibrium if $\mathbf{u}(s) \in \mathbf{P}^{C}\left(s_{N}\right)$ for all $s \in \mathbf{S}$. Here we demonstrate that all profiles are Pareto inferior to the all-T profile when $P \in\left[P_{0}^{*}, 1\right]$. Under this condition, a shift from the all-T to any profile maintains the payoff vector or decreases at least one player's payoff. The $i$-th player's profile can change in four ways (Table 3 ).

First, similar to (10),

$$
\mathbf{u}\left(s_{N}\right) \in \mathbf{P}^{C}\left(s_{N}\right)
$$

Second, every degree free-rider profile is Pareto inferior to the all-T profile. When the players shift from $s_{N}$ to $s_{k}, k$-players are type S10a, and other players are type $\mathrm{S} 10 \mathrm{~b}$. Since $U_{i}(\mathrm{~T}, N-1)>U_{i}(\mathrm{~T}, k-1)$, the shift from $s_{N}$ to $s_{k}$ decreases at least one player's payoff:

$$
\mathbf{u}\left(s_{k}\right) \in \mathbf{P}^{C}\left(s_{N}\right), \forall k \in \mathbf{K}, \forall s_{k} \in \mathbf{S}_{k} .
$$

Third, the all-F profile is Pareto inferior to the all-T profile if $P \in\left[P_{0}^{*}, 1\right]$. When the players shift from $s_{N}$ to $s_{0}$, every player is type S9. The profile shift maintains the payoff vector or decreases every player's payoff if $U_{i}(\mathrm{~T}, N-1) \geq$ $U_{i}(\mathrm{~F}, 0)$. From this inequality,

$$
\mathbf{u}\left(s_{0}\right) \in \mathbf{P}^{C}\left(s_{N}\right) \leftrightarrow P \in\left[P_{0}^{*}, 1\right]
$$


All profiles are Pareto inferior to the all-T profile under the condition (21).

The conditions for Pareto efficient Nash equilibria (PNE) and social dilemmas (SDs) are listed in Table 4.

\section{Results}

\subsection{Individual and social rationality in ITN misuse}

The distribution of PNE and SDs suggests that ITN use for alternative purposes can be an individually and socially rational strategy (Fig. 2).

ITN misuse can be an individually rational strategy when the probability of malaria infection $(P)$ is sufficiently low. If the infection probability is lower than $P_{0}$, the malaria infection burden can be offset by extra income from ITN misuse. As a result, every player is attracted to ITN misuse, and the all-F Nash equilibrium holds. If the infection probability is higher than $P_{0}$, at least one player changes its strategy from $\mathrm{F}$ to $\mathrm{T}$, and starts ITN use for malaria prevention. At the free-rider Nash equilibrium, improper ITN users free ride on the community effect provided by proper ITN users. As the infection probability increases, the number of proper ITN users (degree $k$ ) increases, and the number of free riders decreases. When the infection probability is higher than $P_{N-1}$, the malaria infection burden is heavy, and hence ITN use for malaria prevention is attractive for the players. Every player is attracted to the strategy T, and the all-T Nash equilibrium holds.

ITN misuse can be also a socially rational strategy. When the infection probability is lower than $P_{0}^{*}$, the all-F Nash equilibrium is Pareto efficient. Since the benefit from ITN misuse exceeds the malaria infection burden, 
change in the strategy from $\mathrm{F}$ to $\mathrm{T}$ decreases the player's payoff. If the infection probability lies between $P_{0}^{*}$ and $P_{0}$, the all-T profile is Pareto efficient. However, the players are attracted to the all-F profile, and face with a social dilemma. The $k$-th degree free-rider Nash equilibrium is divided into the PNE and SD at the border $P=P_{k}^{*}$. $P_{k}^{*}$ approaches $P_{k}$ as the degree $k$ approaches $N-1$. It follows that the $(N-1)$-th degree free-rider Nash equilibrium is always Pareto efficient except at the right side edge $P=P_{N-1}$. At a free-rider PNE, free riders can gain a higher payoff than at the all-T profile. In general, individually rational free riders hamper social rationality $[13,20,21]$. However, free riders in the mosquito net game can contribute to social rationality. Note that the all-T Nash equilibrium is always Pareto efficient.

\subsection{ITN misuse driven by poverty}

ITN misuse is associated with poverty measured by low labor productivity. Labor productivity has no direct impact on the distribution of Nash and Pareto equilibria (Table 4), but indirectly influences it through the misuse effect $(\beta)$. Let $\Delta L \in[0, \infty)$ (\$/capita) be extra income from ITN use for alternative purposes. Assume that $\Delta L$ is independent of labor productivity $(L)$. The misuse effect is the rate of payoff increase from ITN misuse. We have

$$
\beta:=\frac{L+\Delta L}{L}=1+\frac{\Delta L}{L} \propto L^{-1} .
$$

This means that the misuse effect is proportional to the inverse of labor productivity. Low-income and high-income communities are characterized by high and low values of $\beta$, respectively. 
Each player's decision making changes as the misuse effect increases (Fig. 3). When the misuse effect is weak (i.e., $\beta \approx 1$ ), ITN use for malaria prevention is individually rational, and the all- $\mathrm{T}$ Nash equilibrium holds regardless of malaria infection probability $(P)$. As $\beta$ increases, extra income from ITN misuse increases, and hence the strategy F can be individually rational. When the misuse effect is sufficiently strong (i.e., $\beta \gg 1$ ), ITN misuse is individually rational, and the all-F and free-rider Nash equilibria hold regardless of $P$.

In a high-income community, the extra income $\Delta L$ is negligible compared with each player's labor productivity, and hence the misuse effect is weak. Since the benefit from ITN misuse cannot offset the malaria infection burden, every player uses ITNs for malaria prevention even if the infection probability is low (Fig. 4a). The all-T Nash equilibrium is always Pareto efficient, and proper ITN use also satisfies social rationality. In a low-income community, however, the extra income $\Delta L$ is attractive for the players with low labor productivity, and the misuse effect is strong. As a result, at least one player uses ITNs to enhance its labor productivity even if the infection probability is high (Fig. 4b, see also Appendix B). ITN misuse is not always socially rational, and the players may face with a social dilemma. Thus, poverty is a major reason for alternative ITN use.

\subsection{Human community size and decision making}

The distribution of Nash equilibrium depends on human community size (the number of players) in addition to mosquito net effects. As shown by (B.1) in Appendix B, the maximum number of proper ITN users $\left(k_{\max }\right)$ is limited by the individual, community, and misuse effects. For example, we 
consider the community in which the parameters $\alpha_{1}, \alpha_{2}$, and $\beta$ are 0.6, 0.99, and 1.5, respectively (Fig. 5). The community has $k_{\max }=60$. If the community contains 50 players, the all-T Nash equilibrium is achieved when the probability of malaria infection is sufficiently high. If the community contains 100 players, however, the free-rider Nash equilibria from 61st to 99th degree and the all-T Nash equilibrium are crowded out of the domain $P \in[0,1]$. This result means that at most 60 players use ITNs for malaria prevention even when the infection probability is extremely high (e.g., $P=1$ ). The achievement of the all-T Nash equilibrium would become more difficult as the number of players increases.

\subsection{Economic regulation of ITN misuse}

From the viewpoint of public health, it is desirable that all players use ITNs for malaria prevention. However, the misuse of ITNs can be an individually and socially rational strategy. This mismatch between public-health and economic benefits is interpreted as a social dilemma. When the players are attracted to the all-F and free-rider Nash equilibria, they cannot reach the all-T Nash equilibrium without an incentive to stop ITN misuse. The incentive can be created by imposing a tax on extra income from ITN misuse. A tax is a major economic tool for dissolving social dilemmas which appear in the voluntary provision of public goods $[13,21]$ and the shared use of ecosystem services [20].

Here we consider the situation where the players with strategy $\mathrm{F}$ are forced to pay a tax. Let $r \in[0,1]$ be the tax rate. The expected payoff 
inclusive of tax is given by

$$
U_{i}^{\operatorname{tax}}\left(\sigma_{i}, m_{-i}\right):= \begin{cases}U_{i}\left(\sigma_{i}, m_{-i}\right) & \left(\sigma_{i}=\mathrm{T}\right) \\ (1-r) U_{i}\left(\sigma_{i}, m_{-i}\right) & \left(\sigma_{i}=\mathrm{F}\right)\end{cases}
$$

The strict all-T Nash equilibrium holds if $U_{i}^{\operatorname{tax}}(\mathrm{T}, N-1)>U_{i}^{\operatorname{tax}}(\mathrm{F}, N-1)$. Solving this inequality with respect to $r$ gives

$$
r>r^{*}:=1-\frac{U_{i}(\mathrm{~T}, N-1)}{U_{i}(\mathrm{~F}, N-1)}
$$

where $r^{*}$ is the rate of payoff increase caused by the shift from the all-T to $(N-1)$-th degree free-rider profile. If $U_{i}(\mathrm{~T}, N-1)>U_{i}(\mathrm{~F}, N-1)$, the all-T Nash equilibrium naturally holds and the tax is unnecessary. If $U_{i}(\mathrm{~T}, N-1) \leq U_{i}(\mathrm{~F}, N-1)$, the players are attracted to the all-T Nash equilibrium at a tax rate $r>r^{*}$. Fig. 6 shows the tax rate $r^{*}$ corresponding to the misuse effect $(\beta)$. In a high-income community characterized by a low $\beta$ (see Eq. (22)), extra income from ITN misuse is negligible, and the all-T Nash equilibrium holds without the tax. In a low-income community characterized by a high $\beta$, extra income from ITN misuse is attractive for the players, and hence a high tax rate is essential to eliminate free riding and to achieve the all-T Nash equilibrium.

\section{Conclusions}

In this paper, we developed an $N$-player mosquito net game to represent decision making of people threatened by malaria, and theoretically showed that the misuse of ITNs is underpinned by individual and social rationality. The $N$-player mosquito net game is an extension of the two-player mosquito 
net game presented by Honjo et al. [8]. Owing to this extension, we could demonstrate that decision making of ITN users is influenced by human community size.

We found that the number of players using ITNs for malaria prevention increases as the probability of malaria infection increases. When all players properly use ITNs, each player's strategy is both individually and socially rational. However, we also found that the misuse of ITNs can be an individually and socially rational strategy. If the benefit from ITN misuse exceeds the malaria infection burden, many players are attracted to ITN misuse even if the infection probability is high.

Alternative ITN users can be interpreted as free riders. Proper ITN users provide the community effect which indirectly protects all players from malaria $[9,7,10]$. By free riding on the community effect, each improper ITN user can decrease its infection probability without abandoning extra income from ITN misuse. Unlike in the voluntary provision of public goods [13, 21], there exist social rational free riders in the mosquito net game. This result arises from the fact that the community effect is not a pure public good. In the "classic" public goods game, free riding is a social rational strategy, but is not chosen by the players because the provision of public goods is always individually irrational. In the mosquito net game, the community effect plays a role similar to public goods, but it can be provided by individually rational players due to the direct benefit from proper ITN use. However, if the number of free riders is excessive at a given infection probability, the community effect is insufficient to offset the malaria infection burden, and the players would face with a social dilemma. 
The $N$-player game also confirms a key insight from the two-player game [8], that low labor productivity or poverty can be an incentive for the misuse of ITNs. Most of the malaria endemic areas are located in developing countries with low incomes $[18,22]$. Extra income from alternative ITN use, which is negligible for high-income players, is attractive for low-income players. Low-income players in malaria endemic areas use ITNs to enhance their labor productivity, i.e., to improve their pauper condition via an income increase. This result indicates that adherence to ITN use in malaria endemic area is likely influenced by socioeconomic status of ITN users, as suggested by several articles [3].

Decision making of players is also influenced by human community size (the number of players). We found that the number of proper ITN users has the upper limit determined by mosquito net effects. If the number of players is less than the upper limit, all players use ITN for malaria prevention under a sufficiently high infection probability. However, if the number of players is greater than the upper limit, at least one player misuses ITNs even if the infection probability is extremely high. This result suggests that the collective ITN use for malaria prevention becomes more difficult as human community size increases.

Authorities can regulate the misuse of ITNs using a tax system. We showed that ITN use for malaria prevention is promoted by imposing a tax on extra income from ITN misuse. In a low-income community, the benefit from ITN misuse is relatively large, and hence a high tax rate is essential to eliminate free riders.

Our results suggest that the success of ITN distribution depends on how 
people threatened by malaria use ITNs. It follows that malaria control programs need to consider decision making of people in malaria endemic areas.

Most of the malaria deaths are children younger than five years old [22]. It would be fruitful to develop a mosquito net game which covers differences in vulnerability to malaria between adults and children. Thus, a further extension of the mosquito net game is to introduce age structure in the players. 


\section{Acknowledgments}

We would like to thank Noboru Minakawa, Akira Kaneko, and Luis Fernando Chaves for fruitful comments. KH is supported by a fellowship from Japan Society for the Promotion of Science. 


\section{Appendix A.}

The aggregate community effect (ACE) is expressed as a function of per capita community effect $\left(\alpha_{2}\right)$ and the number of proper ITN users $(m)$. In the main text, we used the power functional form $\alpha_{2}^{m}$ in order to simplify equilibrium analysis of the game. However, it is possible to use an alternative functional form. Here we consider the case where the ACE follows the exponential functional form $e^{-m\left(1-\alpha_{2}\right)}$. Under the power functional form, the ACE rapidly increases (i.e., $\alpha_{2}^{m}$ decreases) as $m$ increases. The impact of $m$ on the ACE becomes moderate by using the exponential functional form.

When the ACE follows the exponential functional form, the all-F Nash equilibrium holds if

$$
P \in\left[0, \hat{P}_{0}\right], \quad \hat{P}_{0}:=\frac{\beta-1}{\beta-\alpha_{1} e^{-\left(1-\alpha_{2}\right)}} .
$$

The all-T Nash equilibrium holds if

$$
P \in\left[\hat{P}_{N-1}, 1\right] \cap[0,1], \quad \hat{P}_{N-1}:=\frac{\hat{P}_{0}}{e^{-(N-1)\left(1-\alpha_{2}\right)}} .
$$

The free-rider Nash equilibrium holds if $P \in\left[\hat{P}_{0}, \hat{P}_{N-1}\right] \cap[0,1]$. We compared this distribution of Nash equilibria with that derived from the power functional form (see also Section 3.1). Within the parameter region spanned by $\alpha_{1} \in(0,1), \alpha_{2} \in[0.95,1), \beta \in(1,2]$, and $N=100$, the following inequalities hold: $\left|\hat{P}_{0}-P_{0}\right|<0.01$ and $\left|\min \left\{\hat{P}_{N-1}, 1\right\}-\min \left\{P_{N-1}, 1\right\}\right|<0.12$. This result indicates that the difference between the two distributions is small. 


\section{Appendix B.}

Fig. $4 \mathrm{~b}$ is drawn based on the following calculation. First, the maximum degree of free-rider Nash equilibria is given by

$$
k_{\max }:=\text { floor }\left(1+\frac{\log P_{0}}{\log \alpha_{2}}\right), P_{0}=\frac{\beta-1}{\beta-\alpha_{1} \alpha_{2}},
$$

where floor $(x)$ gives the largest integer not greater than $x$. (B.1) is obtained by solving the condition $P_{k-1} \in[0,1]$ with respect to degree $k$. Substituting the parameters $\alpha_{1}=0.6, \alpha_{2}=0.99$, and $\beta=1.5$ into (B.1) gives $k_{\max }=60$. It follows that free-rider Nash equilibria with degrees higher than 60 and the all-T Nash equilibrium are crowded out of the domain $P \in[0,1]$.

Second, the minimum degree of free-rider PNE is given by

$$
\begin{gathered}
k_{\min }^{*}:=\operatorname{ceiling}\left(N+1-\frac{\log \left(\alpha_{2}-\left(1-\alpha_{2}\right) \beta / \alpha_{1}\right)}{\log \alpha_{2}}\right), \\
\beta<\frac{\alpha_{1} \alpha_{2}}{1-\alpha_{2}},
\end{gathered}
$$

where ceiling $(x)$ gives the smallest integer not less than $x$. (B.2) is obtained by solving the condition $P_{k}^{*} \in\left[P_{k-1}, P_{k}\right]$ with respect to degree $k$. Substituting the above parameters and $N=100$ into (B.2) gives $k_{\min }^{*}=98$. This means that only the 98th and 99th degree free-rider Nash equilibria can be Pareto efficient. All the free-rider Nash equilibria contained by the domain $P \in[0,1]$, which have degrees lower than or equal to 60 , are Pareto inefficient (i.e., social dilemmas). Note that every degree free-rider Nash equilibrium can be Pareto efficient if $\beta \geq \alpha_{1} \alpha_{2} /\left(1-\alpha_{2}\right)$. 


\section{References}

[1] Baume, C. A., Reithinger, R., Woldehanna, S., 2009. Factors associated with use and non-use of mosquito nets owned in Oromia and Amhara Regional States, Ethiopia. Malaria Journal 8, 264.

[2] Chaves, L. F., Kaneko, A., Taleo, G., Pascual, M., Wilson, M. L., 2008. Malaria transmission pattern resilience to climatic variability is mediated by insecticide-treated nets. Malaria Journal 7, 100.

[3] Chaves, L. F., Koenraadt, C. J. M., 2010. Climate change and highland malaria: fresh air for a hot debate. The Quarterly Review of Biology 85, $27-55$.

[4] Dawes, R. M., 1980. Social dilemmas. Annual Review of Psychology 31, 169-193.

[5] Dumitrescu, D., Lung, R. I., Gaskó, N., 2011. Detecting strong Berge Pareto equilibrium in a non-cooperative game using an evolutionary approach. 6th IEEE International Symposium on Applied Computational Intelligence and Informatics, 101-104.

[6] Fegan, G. W., Noor, A. M., Akhwale, W. S., Cousens, S., Snow, R. W., 2007. Effect of expanded insecticide-treated bednet coverage on child survival in rural Kenya: a longitudinal study. Lancet 370, 1035-1039.

[7] Hawley, W. A., Phillips-Howard, P. A., ter Kuile, F. O., Terlouw, D. J., Vulule, J. M., Ombok, M., Nahlen, B. L., Gimnig, J. E., Kariuki, S. K., Kolczak, M. S., Hightower, A. W., 2003. Community-wide effects of 
permethrin-treated bed nets on child mortality and malaria morbidity in western Kenya. The American Journal of Tropical Medicine and Hygiene $68,121-127$.

[8] Honjo, K., Chaves, L. F., Satake, A., Kaneko, A., Minakawa, N., 2013. When they don't bite, we smell money: understanding malaria bednet misuse. Parasitology 140, 580-586.

[9] Howard, S. C., Omumbo, J., Nevill, C., Some, E. S., Donnelly, C. A., Snow, R. W., 2000. Evidence for a mass community effect of insecticidetreated bednets on the incidence of malaria on the Kenyan coast. Transactions of the Royal Society of Tropical Medicine and Hygiene 94, 357360.

[10] Killeen, G. F., Smith, T. A., Ferguson, H. M., Mshinda, H., Abdulla, S., Lengeler, C., Kachur, S. P., 2007. Preventing childhood malaria in Africa by protecting adults from mosquitoes with insecticide-treated nets. PLoS Medicine 4, e229.

[11] Lindblade, K. A., Eisele, T. P., Gimnig, J. E., Alaii, J. A., Odhiambo, F., ter Kuile, F. O., Hawley, W. A., Wannemuehler, K. A., PhillipsHoward, P. A., Rosen, D. H., Nahlen, B. L., Terlouw, D. J., Adazu, K., Vulule, J. M., Slutsker, L., 2004. Sustainability of reductions in malaria transmission and infant mortality in western Kenya with use of insecticide-treated bednets. The Journal of the American Medical Association 291, 2571-2580.

[12] Lover, A. A., Sutton, B. A., Asy, A. J., Wilder-Smith, A., 2011. An ex- 
ploratory study of treated-bed nets in Timor-Leste: patterns of intended and alternative usage. Malaria Journal 10, 199.

[13] Marwell, G., Ames, R. E., 1981. Economists free ride, does anyone else? Experiments on the provision of public goods, IV. Journal of Public Economics 15, 295-310.

[14] Minakawa, N., Dida, G. O., Sonye, G. O., Futami, K., Kaneko, S., 2008. Unforeseen misuses of bed nets in fishing villages along Lake Victoria. Malaria Journal 7, 165.

[15] Nash, J. F., 1950. Equilibrium points in n-person games. Proceedings of the National Academy of Sciences of the United States of America 36, $48-49$.

[16] Nash, J. F., 1951. Non-cooperative games. Annals of Mathematics 54, 286-295.

[17] Roll Back Malaria Partnership, 2005. RBM Global Strategic Plan 20052015. RBM Partnership Secretariat, Geneva, Switzerland.

[18] Sachs, J., Malaney, P., 2002. The economic and social burden of malaria. Nature 415, 680-685.

[19] Scalzo, V., 2010. Pareto efficient Nash equilibria in discontinuous games. Economics Letters 107, 364-365.

[20] Scheffer, M., Brock, W., Westley, F., 2000. Socioeconomic mechanisms preventing optimum use of ecosystem services: an interdisciplinary theoretical analysis. Ecosystems 3, 451-471. 
[21] Stiglitz, J. E., Walsh, C. E., 2002. Economics, 3rd Edition. W.W. Norton \& Company, New York, U.S.

[22] World Health Organization, 2012. World Malaria Report 2012. World Health Organization, Geneva, Switzerland. 
Table 1: Changes in the $i$-th player's profile with a shift from the all-F to any profile. S1: Shift to the all-F profile itself. S2: Shifts to the $k$-th degree free-rider profile. S3: Shift to the all-T profile.

\begin{tabular}{ll}
\hline Type & Change in the $i$-th player's profile \\
\hline S1 & $(\mathrm{F}, 0) \Rightarrow(\mathrm{F}, 0)$ \\
\hline S2a & $(\mathrm{F}, 0) \Rightarrow(\mathrm{T}, k-1)$ \\
S2b & $(\mathrm{F}, 0) \Rightarrow(\mathrm{F}, k)$ \\
\hline S3 & $(\mathrm{F}, 0) \Rightarrow(\mathrm{T}, N-1)$ \\
\hline
\end{tabular}


Table 2: Changes in the $i$-th player's profile with a shift from the $k$-th degree free-rider to any profile. S4: Shifts to the $k$-th degree free-rider profile. S5: Shifts to the all-F profile. S6: Shifts to the $k_{0}$-th degree free-rider profiles; $k_{0} \in\{1,2, \ldots, k-1\}$. S7: Shifts to the $k_{1}$-th degree free-rider profiles; $k_{1} \in\{k, k+1, \ldots, N-1\}$. S8: Shifts to the all-T profile.

\begin{tabular}{ll}
\hline Type & Change in the $i$-th player's profile \\
\hline S4a & $(\mathrm{T}, k-1) \Rightarrow(\mathrm{T}, k-1)$ \\
S4b & $(\mathrm{T}, k-1) \Rightarrow(\mathrm{F}, k)$ \\
S4c & $(\mathrm{F}, k) \Rightarrow(\mathrm{T}, k-1)$ \\
S4d & $(\mathrm{F}, k) \Rightarrow(\mathrm{F}, k)$ \\
\hline S5a & $(\mathrm{T}, k-1) \Rightarrow(\mathrm{F}, 0)$ \\
S5b & $(\mathrm{F}, k) \Rightarrow(\mathrm{F}, 0)$ \\
\hline S6a & $(\mathrm{T}, k-1) \Rightarrow\left(\mathrm{T}, k_{0}-1\right)$ \\
S6b & $(\mathrm{T}, k-1) \Rightarrow\left(\mathrm{F}, k_{0}\right)$ \\
S6c & $(\mathrm{F}, k) \Rightarrow\left(\mathrm{T}, k_{0}-1\right)$ \\
S6d & $(\mathrm{F}, k) \Rightarrow\left(\mathrm{F}, k_{0}\right)$ \\
\hline S7a & $(\mathrm{T}, k-1) \Rightarrow\left(\mathrm{T}, k_{1}-1\right)$ \\
S7b & $(\mathrm{T}, k-1) \Rightarrow\left(\mathrm{F}, k_{1}\right)$ \\
S7c & $(\mathrm{F}, k) \Rightarrow\left(\mathrm{T}, k_{1}-1\right)$ \\
S7d & $(\mathrm{F}, k) \Rightarrow\left(\mathrm{F}, k_{1}\right)$ \\
\hline S8a & $(\mathrm{T}, k-1) \Rightarrow(\mathrm{T}, N-1)$ \\
S8b & $(\mathrm{F}, k) \Rightarrow(\mathrm{T}, N-1)$ \\
\hline
\end{tabular}


Table 3: Changes in the $i$-th player's profile with a shift from the all-T to any profile. S9: Shift to the all-F profile. S10: Shifts to the $k$-th degree free-rider profile. S11: Shift to the all-T profile itself.

\begin{tabular}{ll}
\hline Type & Change in the $i$-th player's profile \\
\hline S9 & $(\mathrm{T}, N-1) \Rightarrow(\mathrm{F}, 0)$ \\
\hline S10a & $(\mathrm{T}, N-1) \Rightarrow(\mathrm{T}, k-1)$ \\
S10b & $(\mathrm{T}, N-1) \Rightarrow(\mathrm{F}, k)$ \\
\hline S11 & $(\mathrm{T}, N-1) \Rightarrow(\mathrm{T}, N-1)$ \\
\hline
\end{tabular}


Table 4: Conditions for Pareto efficient Nash equilibria (PNE) and social dilemmas (SDs). $\varnothing$ denotes the empty set. $P_{0}:=(\beta-1) /\left(\beta-\alpha_{1} \alpha_{2}\right)$, $P_{k}:=P_{0} / \alpha_{2}^{k}, P_{0}^{*}:=(\beta-1) /\left(\beta-\alpha_{1} \alpha_{2}^{N}\right), P_{k}^{*}:=(\beta-1) /\left(\alpha_{2}^{k} \beta-\alpha_{1} \alpha_{2}^{N}\right)$, $k \in\{1,2, \ldots, N-1\}$.

\begin{tabular}{ll}
\hline Equilibrium & Condition \\
\hline All-F PNE & $P \in\left[0, P_{0}^{*}\right]$ \\
$k$-th degree free-rider PNE & $P \in\left[P_{k-1}, P_{k}^{*}\right) \cap[0,1]$ \\
All-T PNE & $P \in\left[P_{N-1}, 1\right]$ \\
\hline All-F SD & $P \in\left(P_{0}^{*}, P_{0}\right]$ \\
$k$-th degree free-rider SD & $P \in\left[P_{k}^{*}, P_{k}\right] \cap[0,1]$ \\
All-T SD & $P \in \varnothing$ \\
\hline
\end{tabular}


(a)

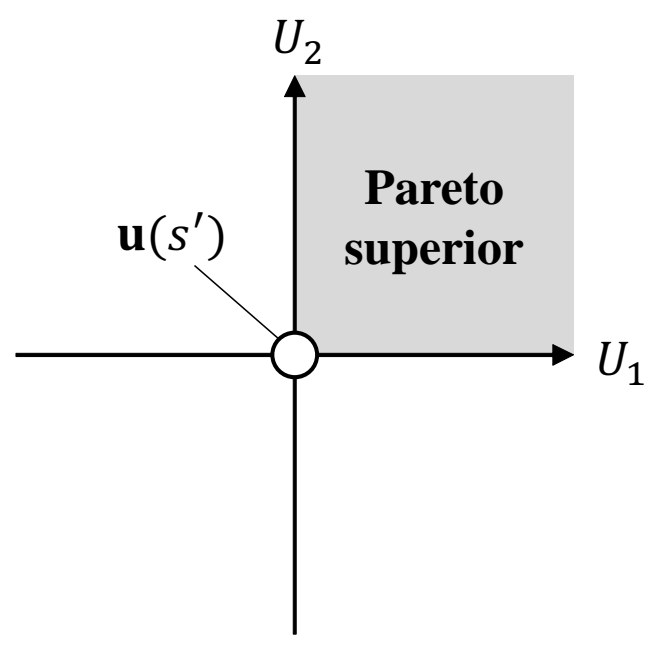

(b)

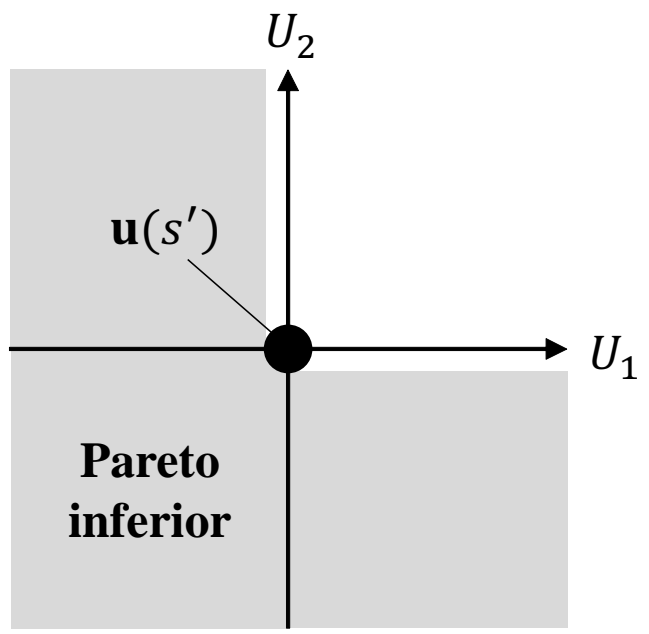

Fig. 1. Pareto superior and inferior regions to a payoff vector $\mathbf{u}\left(s^{\prime}\right)$ $(N=2)$. If the players move from $\mathbf{u}\left(s^{\prime}\right)$ to the Pareto superior region, at least one player can increase its payoff without decreasing another player's payoff. The move from $\mathbf{u}\left(s^{\prime}\right)$ to the Pareto inferior region maintains the payoff vector or decreases at least one player's payoff. 


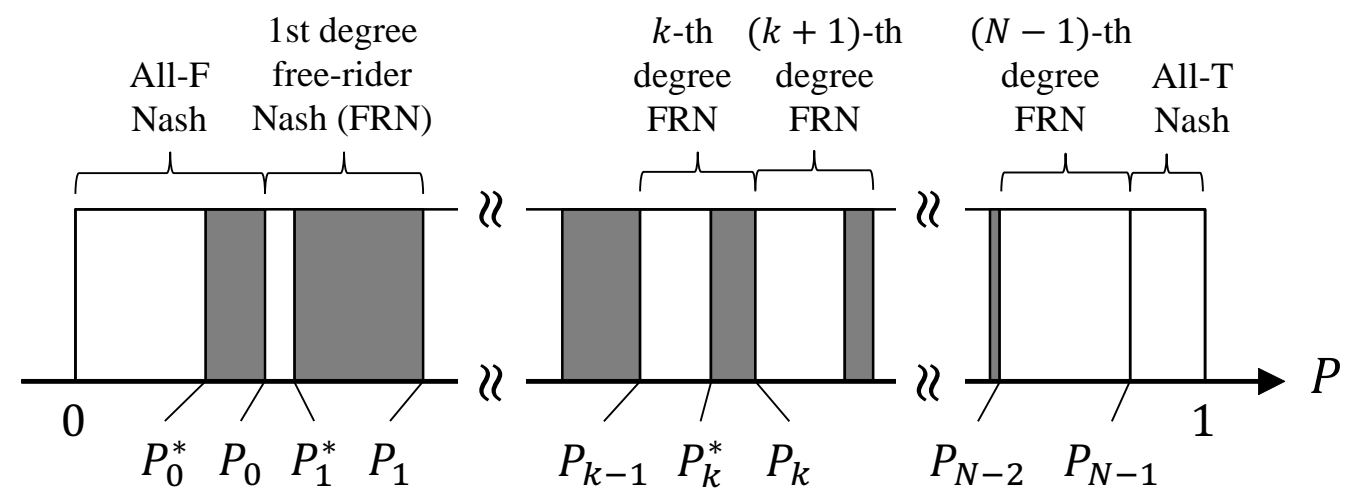

Fig. 2. Distribution of Pareto efficient Nash equilibria (PNE) and social dilemmas (SDs) corresponding to the probability of malaria infection $(P)$. The degree $k \in\{1,2, \ldots, N-1\}$ is the number of players with strategy $\mathrm{T}$ (ITN use for malaria prevention), which increases as $P$ increases. As $k$ approaches $N-1, P_{k}^{*}$ approaches $P_{k}$. The $(N-1)$-th degree free-rider Nash equilibrium is Pareto efficient except at the right side edge $P_{N-1}$. The all-T Nash equilibrium is always Pareto efficient. 


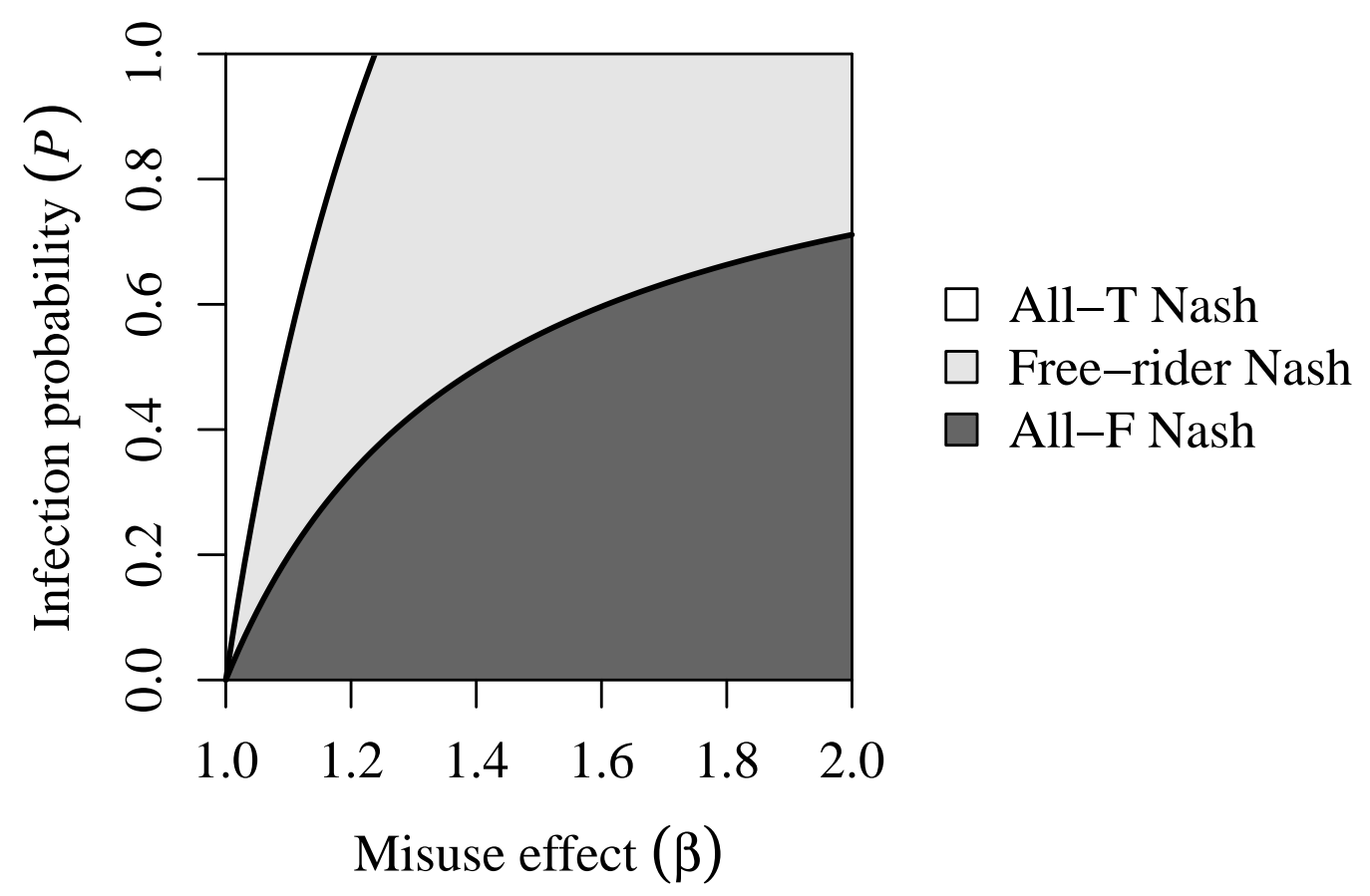

Fig. 3. Distribution of Nash equilibria corresponding to the misuse effect $\left(\alpha_{1}=0.6, \alpha_{2}=0.99, N=100\right)$. 
(a) High-income community $(\beta=1.01)$

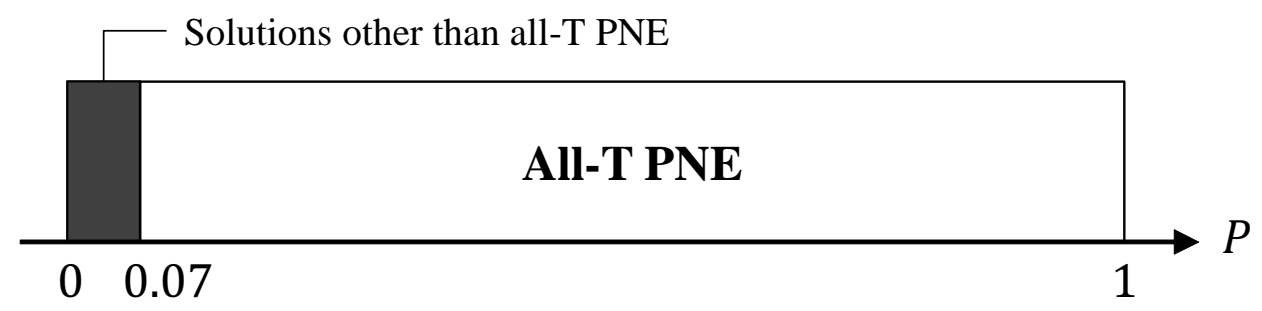

(b) Low-income community ( $\beta=1.50)$

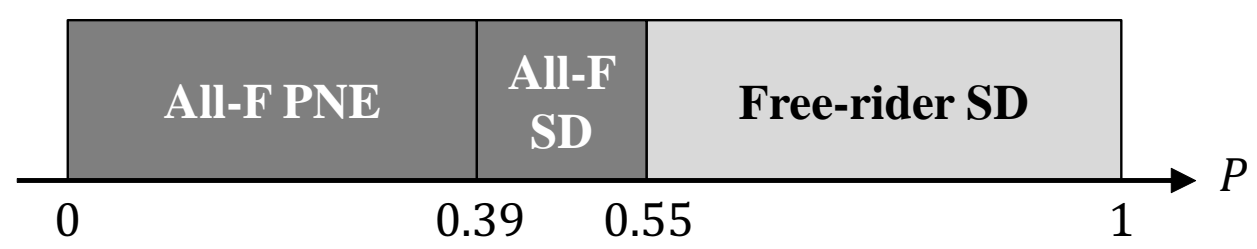

Fig. 4. Pareto efficient Nash equilibria (PNE) and social dilemmas (SDs) in high-income and low-income communities $\left(\alpha_{1}=0.6, \alpha_{2}=0.99\right.$, $N=100)$. Fig. 4b is drawn based on the calculation shown in Appendix B. 


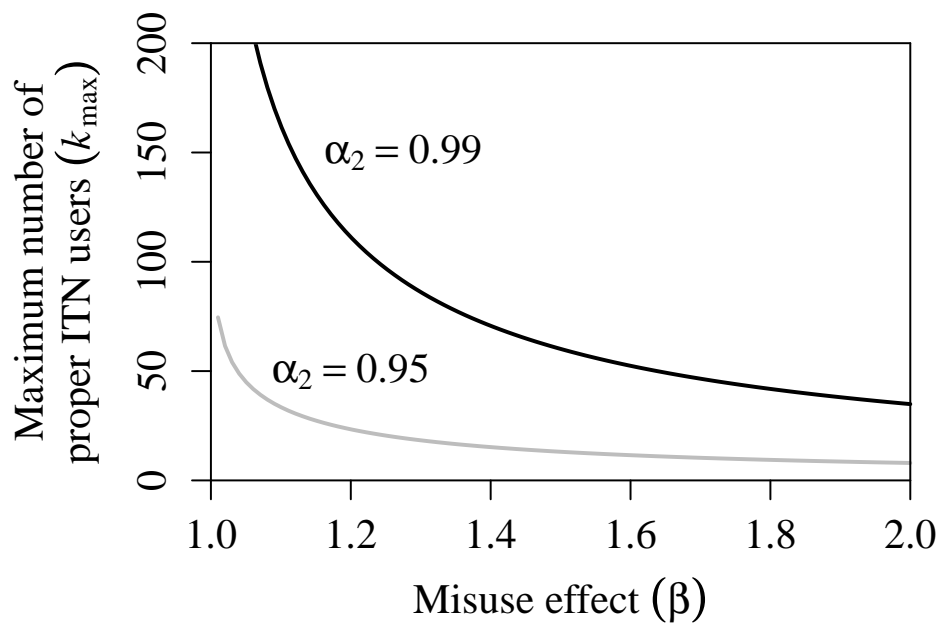

Fig. 5. Maximum number of proper ITN users $\left(k_{\max }\right)$ corresponding to mosquito net effects. The individual effect $\left(\alpha_{1}\right)$ is fixed at $0.6 . k_{\max }$ is given by (B.1) in Appendix B. If the number of players is less than $k_{\max }$, the all-T Nash equilibrium is achieved under a sufficiently high infection probability $(P)$. If the number of players is greater than $k_{\max }$, at least one player misuses ITNs even when $P=1$. 


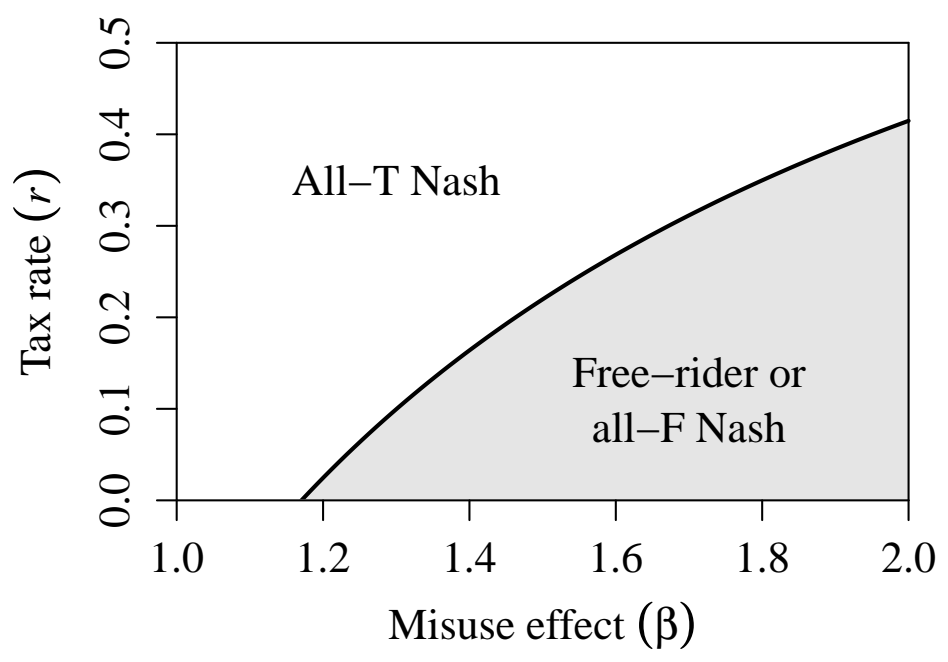

Fig. 6. Tax rate curve between the all-T and free-rider Nash equilibria $\left(\alpha_{1}=0.6, \alpha_{2}=0.99, P=0.8, N=100\right)$. On the tax rate curve, the all-T and $(N-1)$-th degree free-rider Nash equilibria hold simultaneously. 